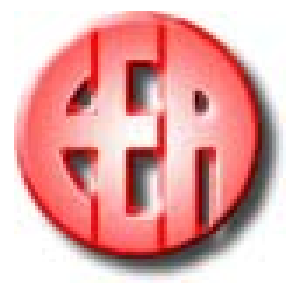

\section{Compte-rendu des Journées électrotechnique du Club EEA 2006}

Gif-sur-Yvette, 15-16 mars 2006

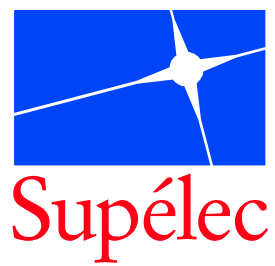

Les XXèmes journées de la section électrotechnique du club EEA ont été organisées les 15 et 16 mars 2006 par le département Energie de Supélec sur le campus de Gif-sur-Yvette.

Cette édition a rassemblé 76 participants, répartis selon le diagramme ci-contre, autour du thème «l'ouverture des marchés de l'electricité ». Ce thème était développé par des interventions d'acteurs industriels (gestionnaire de réseau, producteurs, constructeurs), commerciaux (powernext), institutionnels (CRE) et académiques (universités paris XI et Paris I, Supélec, LEG, L2EP), permettant ainsi de dresser un panorama complet de l'état d'ouverture et des perspectives à venir dans le marché

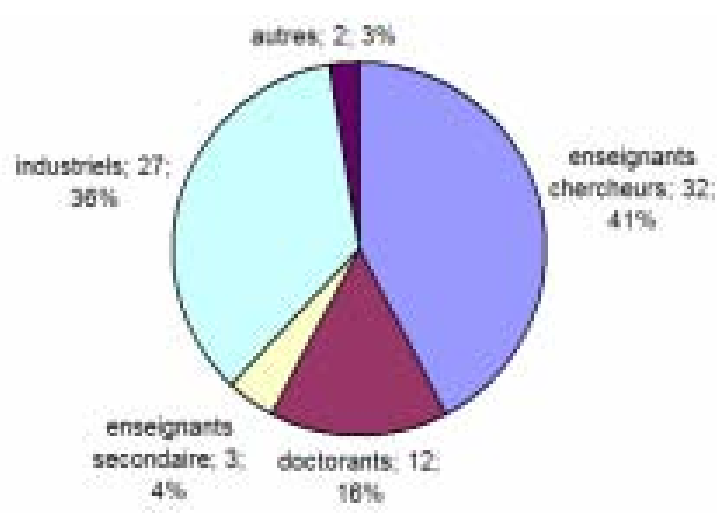
de l'électricité. Le thème choisi est d'actualité, et cette réorganisation du secteur implique de profonds changements sur l'enseignement de l'électrotechnique, dans le sens ou elle modifie complètement l'organisation et les contraintes extérieures du système électrique.

Les journées se sont déroulées en quatre sessions suivies d'une table ronde sur l'enseignement.

J.-M. Glachant, professeur d'économie à Paris XI a ainsi présenté l'état d'ouverture du marché français de l'électricité, et la manière dont la concurrence parvient à rogner le monopole de l'opérateur historique, en espérant que le prochain renouvellement du parc de production amène une meilleure répartition des parts de marché. En attendant, une meilleure intégration des marchés européens, telle que l'a présentée J. Boucher, directeur de la stratégie de Suez Electrabel, permettrait d'introduire plus de concurrence. Celle-ci est d'ores et déjà facilitée par le fonctionnement d'une bourse de l'électricité, dont les rouages nous ont été présentés par A. Mahuet de Powernext. Cette évolution du marché permet de révéler des prix de marché de l'électricité, dont la caractérisation nécessite des modèles aboutis, tels que ceux que nous a présenté $\mathrm{B}$. Vignal, chef de projet chez EDF R\&D. Ces interventions sont à mettre en regard de celle de M. Begovic, professeur d'électrotechnique à Georgia Tech, sur l'état des lieux et le retour d'expérience aux états unis, où la libéralisation est plus avancée.

Cette ouverture à la concurrence amène un certain nombre de contraintes technicoéconomiques à prendre en compte dans l'exploitation du système électrique.

Par exemple les relations entre le transporteur (RTE) et les producteurs ont du être redéfinies, notamment en ce qui concerne les services système. Dorénavant des prescriptions légales 
régissent la fourniture de ces services, et RTE a développé un mécanisme de contrôle des performances vis-à-vis de ces services, que nous a présenté T. Margotin, ingénieur chez RTE. Il en est de même pour la qualité de l'énergie, comme nous l'a présenté X. Mamo, chef de groupe chez EDF R\&D.

En ce qui concerne les interconnexions, qui font dorénavant l'objet de convoitise pour les échanges d'énergie entre les différents marchés nationaux alors qu'elles ont été développées pour permettre une plus grande fiabilité du système et pour des échanges ponctuels entre pays. Ces lignes étant donc souvent saturées, la gestion de l'accès à du être redéfinie, avec une allocation faisant une part de plus en plus grande aux enchères. Parmi les perspectives d'avenir présentées, une allocation des transits et de leurs conséquences (pertes et congestion) permettrait de fournir des signaux économiques permettant aux acteurs d'internaliser des coûts actuellement supportés par l'ensemble des acteurs sans responsabilisation. Par ailleurs C. Staropoli, maître de conférence à Paris I, nous a montré que l'application de l'économie expérimentale au marché de l'électricité permettait de tester de nouvelles règles de marché avant leur mise en œuvre réelle.

Si les nouvelles règles économiques introduisent les principaux changements dans le fonctionnement du système électrique, les évolutions technologiques auront eux aussi un impact fort : selon J.-P. Hutin, directeur des programmes de production chez EDF R\&D, les centrales à gaz aujourd'hui et le nucléaire de nouvelle génération dans un futur proche constitueront une grande part des investissements en moyen de production. Néanmoins, B. Robyns, professeur d'électrotechnique et chercheur au L2EP a montré que la montée en puissance de l'éolien nécessite de redéfinir certains mécanismes pour la prise ne compte de l'aléa de production. Dans un futur un peu moins proche, l'organisation en centrales virtuelles telles que nous les ont présentées C. Kieny, ingénieur d'EDF détaché au LEG offre des perspectives intéressantes en matière d'énergie renouvelable et de production décentralisée. Enfin il ne faut pas oublier les innovations apportés au matériel de transport, tels que les FACTS ou les transformateurs déphaseurs, qui permettent un meilleur contrôle du réseau de transport.

La table ronde sur l'enseignement a permis de faire le point sur les enseignements actuels en électrotechnique, et notamment sur les réseaux d'énergie. Il apparaît que l'économie se fait une place grandissante dans cette spécialité, sans pour autant minimiser les aspects techniques qui conservent une place prédominante dans toutes ces problématiques. 